\title{
Post-Error Behavioral Adjustments Are Facilitated by Activation and Suppression of Task-Relevant and Task-Irrelevant Information Processing
}

\author{
Joseph A. King, ${ }^{1}$ Franziska M. Korb, ${ }^{1}$ D. Yves von Cramon, ${ }^{1,2}$ and Markus Ullsperger ${ }^{2,3}$ \\ ${ }^{1}$ Department of Cognitive Neurology, Max Planck Institute for Human Cognitive and Brain Sciences, 04103 Leipzig, Germany, ${ }^{2}$ Max Planck Research Group \\ Cognitive Neurology, Max Planck Institute for Neurological Research, 50866 Cologne, Germany, ${ }^{3}$ Donders Institute for Brain, Cognition, and Behaviour, \\ Radboud University, 6500 HE Nijmegen, The Netherlands
}

\begin{abstract}
Error monitoring by the posterior medial frontal cortex (pMFC) has been linked to post-error behavioral adaptation effects and cognitive control dynamics in lateral prefrontal cortex (LPFC). It remains unknown, however, whether control adjustments following errors produce post-error behavioral adjustments (PEBAs) by inhibiting inappropriate responses or facilitating goal-directed ones. Here we used functional magnetic resonance imaging to investigate the hemodynamic correlates of PEBAs in a stimulus-response compatibility task. Our task was designed to test whether PEBAs are implemented by suppressing motor responses primed by irrelevant stimulus features (facelocation), redirecting attention to relevant features (face gender), or both or neither of these possibilities. Independent of PEBAs, error-related pMFC activation was followed by post-error recruitment of prefrontal and parietal control regions and, crucially, both (1) suppressed response-related activity in sensorimotor cortex and (2) enhanced target processing in face-sensitive sensory cortex ("fusiform face area"). More importantly, by investigating the covariation between post-error hemodynamic activity and individual differences in PEBAs, we showed that modulation of task-related motor and sensory processing was dependent on whether participants produced generally slower responses ("post-error slowing"; PES) or selectively reduced interference effects ("post-error reduction of interference"; PERI), respectively. Each of these behaviorally dependent effects was mediated by distinct LPFC control mechanisms (PES: inferior frontal junction; PERI: superior frontal sulcus). While establishing relationships between PEBAs and cognitive control, our findings suggest that the neural architecture underlying sequential behavioral adaptation may be determined primarily by how control is executed by the individual when adjustments are needed.
\end{abstract}

\section{Introduction}

The existence of a neural system specialized in error detection and compensation (Gehring et al., 1993) was originally inferred from early behavioral observations that participants performing cognitive tasks often adjust behavior following errors by rapidly "correcting" their responses or responding slower and sometimes more accurately on subsequent trials (Rabbitt, 1966). Over the past two decades, much evidence has accumulated implicating the posterior medial frontal cortex (pMFC) in monitoring performance for erroneous or error-prone actions (Botvinick et al., 2001; Brown and Braver, 2005) and integrating reinforcement history (Holroyd and Coles, 2002; Rushworth et al., 2007) to guide cognitive control mechanisms needed to bias lower-level task-related processing in a goal-directed manner and optimize future action outcomes (Ridderinkhof et al., 2004). Empirical support comes in part from studies that have shown conflict- and error-related pMFC activity to predict trial-by-trial behavioral

Received June 24, 2010; revised July 27, 2010; accepted Aug. 3, 2010.

The assistance of J. Lepsien, G. Lohmann, K. Müller, J. Neumann, and S. Zysset in data analysis is greatly acknowledged. We also thank H. Schmidt-Duderstedt for assistance in preparing the figures.

Correspondence should be addressed to Joseph King, at his present address: Duke University, Center for Cognitive Neuroscience, Box 90999, Durham, NC 27708. E-mail: joe.king@duke.edu.

D0I:10.1523/JNEUROSCI.3274-10.2010

Copyright $\odot 2010$ the authors $\quad 0270-6474 / 10 / 3012759-11 \$ 15.00 / 0$ adaptation effects (Gehring et al., 1993; Debener et al., 2005) and increased recruitment of the lateral prefrontal cortex (LPFC) (Kerns et al., 2004; Cavanagh et al., 2009), a brain region broadly implicated in executing cognitive control (Miller and Cohen, 2001; Tanji and Hoshi, 2008). Complementing these findings, studies have shown sequential behavioral adaptation following conflict to be mediated by controlled processing of task-specific motor (Stürmer et al., 2002) and sensory (Egner and Hirsch, 2005) information. To date, however, evidence linking behavioral adaptation effects following errors to modulation of taskspecific processing has remained elusive. Here we used functional magnetic resonance imaging (fMRI) to explore relationships between the neural mechanisms underlying error monitoring in an interference task, trial-by-trial post-error behavioral adjustments (PEBAs) and, crucially, post-error processing of task-relevant and task-irrelevant information.

PEBAs can be expressed as a generally slower, apparently more cautious response mode ("post-error slowing"; PES) or a selective improvement in interference resolution (post-error reduction of interference; PERI) (Ridderinkhof et al., 2004; Carter and van Veen, 2007). While PES is often assumed to gauge a strategic increase in the motor response threshold to avoid future mistakes (Botvinick et al., 2001; Holroyd et al., 2005), recent evidence suggests that it may merely reflect an unspecific orienting response (Notebaert et al., 2009; Núñez Castellar et al., 2010). Sim- 
ilarly, it is not known whether the PERI effect reflects selective suppression of irrelevant representations (Ridderinkhof, 2002), enhancement of relevant representations, or both.

We investigated the hemodynamic correlates of PEBAs during performance of a face-gender discrimination version of the Simon stimulus-response (S-R) compatibility task (Simon, 1969) (Fig. 1). Efficient accurate performance required featurebased attention to faces while overriding "direct" response activation primed by irrelevant stimulus location (Kornblum et al., 1990; Stürmer et al., 2002). We analyzed the covariation of individual differences in PES and PERI with post-error changes in target processing in the individually defined fusiform face area (FFA) (Kanwisher et al., 1997) and response-related sensorimotor cortex (SMC). Thus, we could assess whether cognitive control adjustments following errors facilitate behavioral adaptation by redirecting attention to relevant sensory input, suppressing the influence of irrelevant information on motor output, or both or neither of these possibilities.

\section{Materials and Methods}

Participants. Twenty-one male volunteers (mean age $=27.9$ years; range $=23-34$ years) participated in this study after giving written informed consent according to institutional guidelines based on the Declaration of Helsinki. All participants were right handed, had normal or corrected-to-normal vision, and reported no history of neurological, psychiatric, or major medical disorder. They were reimbursed with 10 euros for their participation, which lasted $\sim 75 \mathrm{~min}$.

Procedure. Participants performed two tasks during fMRI: a localizer task and the main face-gender classification version of the Simon task (Fig. 1). The purpose of the localizer task was to identify face-sensitive regions of extrastriate cortex (FFA) in which we could use hemodynamic activity during performance of the Simon task as an index to assess whether target processing varied as a function of trial $n-1$ accuracy and/or PEBAs. Stimuli for both tasks were a total of 492 grayscale face portraits (50\% male/ 50\% female) selected from the Picture Database of Morphed Faces (Jäger et al., 2005). Only the original unmorphed images and $100 \%$ morphed faces from the database were used. Localizer task stimuli additionally included 52 face images "scrambled" with Adobe Photoshop (Adobe Systems) to yield distinguishable grid-like patterns, but no human features. All stimuli were rescaled to a size of $185 \times 270$ pixels. For both tasks, stimuli were presented against a black background onto a back-projection screen, which participants viewed in a mirror mounted to the head coil. This setup simulated a monitor viewing distance of $\approx 100 \mathrm{~cm}$, resulting in stimuli subtending $\approx 2.8^{\circ} \times 4.1^{\circ}$ of visual angle. Participants responded to stimuli according to task instructions with left- and right-hand index finger button presses on the left and right buttons of a two-key response device, respectively. Task programming, stimulus presentation and behavioral recording were performed with Presentation software (Neurobehavioral Systems).

During the localizer task, participants observed streams of centrally presented face and scrambled images between interspersed null (fixation) blocks. There were 5 pseudorandomly presented epochs of each event type, each including 15 trials with a fixed intertrial interval of 1000 ms. Images were presented for $200 \mathrm{~ms}$ followed by $800 \mathrm{~ms}$ of fixation. Stimulus epochs included 13 novel images and two 1-back image repetitions. To ensure task engagement, participants were instructed to respond to image repetitions with a right button press.

Immediately following the localizer scan, participants practiced the S-R mapping of the Simon task in a 48-trial simulation block. In each of the 432 trials of the main experiment, a novel male or female face image was presented $(150 \mathrm{~ms})$, either left or right of a permanently present central fixation cross (image center $=3.8^{\circ}$ of visual angle). Stimulus onset occurred at five systematically offset time points [range: $0-1600$ $\mathrm{ms}$; mean interstimulus interval (ISI) $=5000 \mathrm{~ms}$ ] in relation to fMRI acquisition to improve temporal resolution of the blood oxygen leveldependent (BOLD) response. Participants were instructed to rapidly and accurately classify faces according to gender ( $\mathrm{S}-\mathrm{R}$ mapping was counterbalanced across subjects). Faces were presented ipsilateral to the gendermapped response side in half of all trials (compatible trials; COMP) and contralateral to the correct response side in the other (incompatible trials; INCO). Trials occurred in 12 blocks, each including 36 face presentations and 4 pseudorandomly interspersed null events (fixation only). Trial compatibility frequency varied in a blockwise manner: congruent blocks included 70\% COMP/30\% INCO trials, incongruent blocks had $30 \%$ COMP $/ 70 \%$ INCO trials, and balanced blocks were made up of $50 \%$ COMP $/ 50 \%$ INCO trials. Each block type occurred 4 times (in pseudorandomized order across subjects), resulting in equiprobable compatibility sequences across the experiment. To obtain an adequate number of errors for analyses of PEBAs, participants received individually determined performance feedback during brief pauses (10 s) between task blocks. Depending on previous block accuracy rates, verbal feedback encouraged the participant to either "speed up" in the following block (if $<15 \%$ errors were made), "slow down" (if $>25 \%$ errors were made), or "maintain performance quality."

Behavioral data analysis. We investigated PEBAs in reaction time (RT) and error rates after excluding the first trial of each block, as these trials carried no valid information regarding performance sequences. PES was calculated as the difference between mean RTs on correct trials immediately following errors (post-error) versus those following correct responses (correct). PERI was calculated as the difference between S-R compatibility effects (i.e., interference effects; INCO - COMP RT) on correct trials versus those on posterror trials. Previous studies have shown that PEBAs can vary as a function of conflict frequency (Ridderinkhof, 2002) and speed/accuracy instructions (Ullsperger and Szymanowski, 2004; Jentzsch and Leuthold, 2006). Exploratory analyses of the current data did not reveal clear evidence for such variation. However, we did not obtain enough post-error trials for each participant under each of the conflict frequency manipulations and performance feedback conditions to conduct legitimate analyses. Therefore, we focus on experiment-wise variation in PEBAs in the current study.

Image acquisition. Imaging was conducted on a Siemens Trio MR system using a standard head coil. During both tasks, we acquired 28 axial slices positioned parallel to the anterior commissure-posterior commissure (AC-PC) plane (4 mm thickness; $1 \mathrm{~mm}$ gap) covering the whole brain using a single-shot gradient echo-planar imaging (EPI) sequence (Tr: $2000 \mathrm{~ms}$; Te: $30 \mathrm{~ms}$; flip angle: $90^{\circ} ; 64 \times 64$ pixel matrix; field of view: 
$192 \mathrm{~mm}$ ) sensitive to BOLD contrast. Before the functional runs, 2D anatomical modified driven equilibrium Fourier transform (MDEFT) and EPI-T1 slices in plane with the functional data were collected.

Image analysis. Images were first corrected for motion artifacts offline with the Siemens motion correction protocol. All further processing was performed with the software package Leipzig Image Processing and Statistical Inference Algorithms (Lohmann et al., 2001) (http://static.cbs. mpg.de/lipsia/). Slice-time acquisition differences were adjusted using a cubic-spline interpolation algorithm. Baseline signal drifts were removed using a $1 / 100 \mathrm{~Hz}$ temporal high-pass filter and spatial smoothing was applied using a Gaussian filter with $5.65 \mathrm{~mm}$ full width at half maximum (FWHM). To align the functional data slices with a 3D stereotactic coordinate reference system, a rigid linear registration with six degrees of freedom (three rotational and three translational) was performed. The rotational and translational parameters were acquired on the basis of the MDEFT and EPI-T1 slices to achieve an optimal match between these slices and an individual high-resolution 3D reference dataset [MDEFT volume with 160 slices and $1 \mathrm{~mm}$ slice thickness standardized to Talairach stereotactic space (Talairach and Tournoux, 1988)] that was acquired for each subject during a previous scanning session. The rotational and translational parameters were subsequently transformed by linear scaling to a standard size. The resulting parameters were then used to transform the functional slices using trilinear interpolation so that the functional slices were aligned with the coordinate system; generating output data with a spatial resolution of $3 \times 3 \times 3 \mathrm{~mm}\left(27 \mathrm{~mm}^{3}\right)$.

Statistical analysis of both fMRI time series was based on a leastsquares estimation using a general linear regression with prewhitening (Worsley et al., 2002) and performed separately for each participant on voxel-by-voxel basis. For the localizer task data, face, scrambled, and null epochs were each modeled with a boxcar function convolved with a Gaussian hemodynamic response function (HRF). A voxelwise onesample $t$ test (random-effects model) across single-subject face versus scrambled contrast images (i.e., estimates of raw score differences between the specified conditions) was used to identify the group average bilateral FFA (face $>$ scrambled) and, for control purposes, scrambledsensitive regions of extrastriate cortex (scrambled $>$ face). These regions of interest (ROIs) were defined as voxels with $Z$-standardized $t$ values $>3.09$ $(p<0.001)$ belonging to clusters that survived correction for multiple comparisons using a double-thresholding procedure (described in detail below) at an $\alpha$ level of 0.001 . To provide a stronger test of post-error modulation of target processing during the Simon task and/or the variation thereof as a function of PEBAs, we also localized the FFA in each participant. Singlesubject FFA ROIs were identified by searching each participant's face $>$ scrambled $Z$-transformed contrast image for clusters ( $>3$ voxels; $81 \mathrm{~mm}^{3}$ ) with $Z$ values $>2.33$ ( $p<0.01$; uncorrected) within a $15 \mathrm{~mm}$ radius of the peak group average FFA coordinates.

We constructed the design matrix of the Simon task data to estimate the stimulus-locked, trial $n-1$ accuracy-dependent hemodynamic response. Explanatory variables were trials with correct responses preceded by correct trials (correct), incorrect responses preceded by correct trials (error), and correct responses preceded by errors (post-error), respectively convolved with a gamma HRF and its first derivative. Onsets of other events (consecutive errors, omitted responses, and feedback between blocks) were included in the model as regressors of no interest. We additionally modeled each block type with a box-car function (convolved with a Gaussian HRF) to account for potential tonic adaptations. Two linear contrasts were calculated for each participant: error versus correct and post-error versus correct. We conducted two random-effects analyses of the resulting contrast images (one sample $t$ tests) to establish brain regions generally involved in (1) error monitoring and (2) post-error processing in our task (i.e., independent of variability in PEBAs).

Behaviorally informed $f M R I$ analyses. Our motivating hypothesis stated that if PEBAs indeed reflect cognitive control mechanisms (Botvinick et al., 2001; Holroyd and Coles, 2002; Ridderinkhof et al., 2004; Ullsperger and von Cramon, 2004; Holroyd et al., 2005), then they should not only be associated with engagement of brain areas thought to execute control (e.g., LPFC) as previously demonstrated (Garavan et al., 2002; Kerns et al., 2004; Marco-Pallarés et al., 2008; West and Travers, 2008; Cavanagh et al., 2009), but also goal-directed modulation of activity in regions
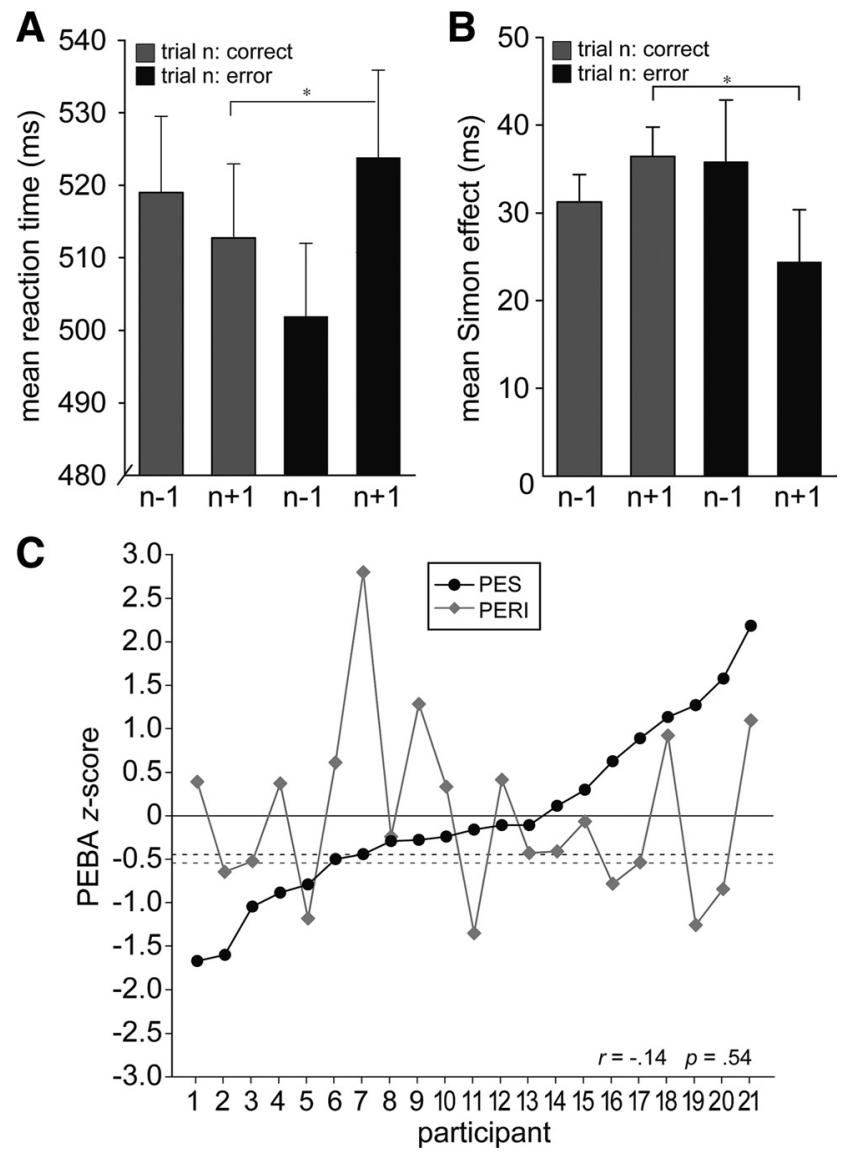

Figure 2. PEBAs. $A$, Mean group RTs ( \pm SEM) on correct trials of the Simon task are depicted as a function of trial $n$ accuracy to illustrate how performance was progressively fast before an error, but generally slowed on post-error trials, constituting PES. ${ }^{*} p<0.05$. $\boldsymbol{B}$, Mean group Simon interference effects (INCO - COMP trial RT; \pm SEM) are shown as in $A$ to illustrate how performance tended to be increasingly influenced by task-irrelevant stimulus location before an error, while post-error performance showed a selective improvement in interference resolution; signifying PERI. ${ }^{*} p<0.05$. C, Individually normalized and Z-standardized (Materials and Methods) degrees of PES (post-error post-correct RT) and PERI (post-correct - post-error interference effect) are plotted for each participant as a function of increasing PES scores across subjects to illustrate the orthogonality of the two measures. Participants with PES Z-values $\geq-0.45$ (dark dashed line) showed numerically slower post-error versus post-correct RTs. Participants with PERI Z-values $\geq-0.54$ (light dashed line) showed numerically reduced post-error interference effects.

specialized in lower-level task-related processing. Further, we assumed that such modulation would vary according to how participants adjusted performance on those trials: generally (as indexed by PES) or specifically (as indexed by PERI). We addressed these hypotheses by incorporating individual degrees of PES and PERI (Fig. $2 C$, compare PES and PERI) as parametric covariates in second-level analyses of the individual posterror $>$ correct contrast images. Thus, we could identify brain regions in which the post-error $>$ correct BOLD signal correlated with individual differences in PEBAs. To explore the hypothesis that PEBAs are triggered by error-related pMFC activity (Botvinick et al., 2001; Holroyd and Coles, 2002; Ridderinkhof et al., 2004; Ullsperger and von Cramon, 2004; Yeung et al., 2004; Holroyd et al., 2005), we also conducted PES- and PERI-informed group analyses of the individual error $>$ correct contrast maps. The PES covariate was calculated for each participant as the $Z$-standardized individual difference between mean post-error and postcorrect RTs in relation to the individual mean RT on all correct trials [(post_error_RT - post_correct_RT)/mean_RT]. The PERI covariate was calculated as the $Z$-standardized individual difference between the mean Simon interference effect (INCO - COMP RT) on the average correct trial and the mean Simon effect on post-error trials in relation to the individual mean Simon effect on all correct trials [(post_correct 
SIMONEFFECT - post_error_SIMONEFFECT)/ mean_SIMONEFFECT].

One participant's standardized PERI score was $>2.5$ SDs from the group mean $(z=2.8)$ and could thus be considered an outlier. To control for undue influence of this participant's data, we conducted all analyses that investigated PERI both under retention and exclusion of this participant's data. Following outlier removal, no significant effects evident in the entire sample became insignificant. Therefore, unless noted otherwise, we report results based on the data of all 21 participants.

To minimize the likelihood of false-positive results in the Simon task data, an initial voxelwise probability threshold was set to $Z>2.58$ ( $p<0.005$; uncorrected). The voxelwise thresholded data were then corrected for multiple comparisons by using cluster-size and mean cluster-value thresholds at significance level of $p<0.05$ as determined by Monte Carlo simulations (1000 iterations), unless noted otherwise.

ROI analyses. To further specify the hemodynamic response in selected a priori and empirically defined ROIs, we sampled the preprocessed stimulus-locked BOLD signal time course for each participant on trials of interest from a 27 voxel cube $\left(729 \mathrm{~mm}^{3}\right)$ centered on group average- and subject-specific (in the FFA) activation maxima, unless noted otherwise. In each region, percentage BOLD signal change was computed for each condition relative to the mean signal intensity across all time steps over the course of the experiment. We determined the maximum signal amplitude occurring in the time window between 3 and $7 \mathrm{~s}$ following stimulation for each participant and submitted the resulting values to appropriate region- and condition-specific statistical analyses.

\section{Results}

\section{Behavioral results}

Overall Simon task performance was characterized by typical interference effects (Kornblum et al., 1990; Simon, 1990; Lu and Proctor, 1995; Proctor and Vu, 2006). Mean RTs for correct responses (excluding correct post-error trials) were slower on INCO trials $(532 \mathrm{~ms})$ than on COMP trials ( $496 \mathrm{~ms} ; t_{(20)}=10.8$; $p<0.0001)$. Likewise, mean INCO trial error rates $(20.4 \%)$ were elevated relative to COMP trial error rates $\left(12.2 \% ; t_{(20)}=\right.$ 6.6; $p<0.0001)$.

The interference effects in error rates (INCO - COMP accuracy), together with the observation that error trial RTs (498 ms) were faster than correct responses $\left(513 \mathrm{~ms} ; t_{(20)}=2.6 ; p<0.05\right)$, suggested that errors were primarily due to insufficient suppression of location-driven "direct" response activation. However, the relatively high COMP trial error rates, in conjunction with the finding that RTs on these trials $(525 \mathrm{~ms})$ were slower than erroneous responses on INCO trials $\left(480 \mathrm{~ms} ; t_{(20)}=4.2 ; p<0.0001\right)$, implied that a portion of errors may have been due to difficulties in deciphering face gender. Regardless of the factors contributing to an error on a given trial, our interest was in how participants might adjust behavior on immediately subsequent trials.

Mean post-error RTs (524 ms) were generally slower than the average correct response in successful performance sequences $\left(513 \mathrm{~ms} ; t_{(20)}=2.2 ; p<0.05\right)$ (Fig. $\left.2 A\right)$, constituting PES. Signifying PERI, mean interference effects (INCO - COMP RT) were selectively reduced on post-error trials relative to those following correct responses $\left(24 \mathrm{~ms}\right.$ vs $\left.36 \mathrm{~ms} ; t_{(20)}=2.5 ; p<0.05\right)$ (Fig. 2 B). The only known study to investigate relationships between PES and PERI found that participants who tended to show PES also tended to have larger post-error interference effects (i.e., less PERI) (Carp and Compton, 2009). Here, however, consistent with the proposal that PES and PERI may reflect distinct forms of PEBAs (Ridderinkhof et al., 2004), we observed no relationship between the two measures across participants $(r=-0.142 ; p=$ $0.54)$. In other words, participants who showed a high degree of PES did not necessarily show a high or low degree of PERI or vice versa (Fig. 2C).

Although error rates did not change on post-error trials [mean error rate on post-error trials $(17.6 \%)$ versus error rate following correct trials $(16.3 \%) ; t_{(20)}=1.3 ; p>0.1$ ], we were interested in whether either form of PEBA might be associated with improved accuracy on post-error trials (Rabbitt, 1966; Laming, 1968; Botvinick et al., 2001; Marco-Pallarés et al., 2008). To this end, we calculated the correlation between (1) the individual difference in error rates following correct trials versus those following errors and (2) the respective individual PES and PERI values. Results indicated that PES was not associated with any change in accuracy on post-error trials $(r=0.1 ; p>0.5)$, but participants who showed greater PERI also showed a tendency to repeat their errors $(r=-0.34 ; p>0.09)$. However, this trend diminished under exclusion of the participant whose PERI value could have been considered an outlier $(r=-0.28 ; p=0.23)$ (Fig. $2 C$, participant 7; see also Materials and Methods). Thus, it is unlikely that the post-error RT adjustments investigated here reflected a shift in performance favoring accuracy over speed or vice versa.

\section{fMRI results: error monitoring}

An initial analysis of the fMRI data established an expected pattern of error $>$ correct pMFC activation (peak Talairach coordinates: $x=1, y=17, z=42 ; 11,313 \mathrm{~mm}^{3} ; Z_{\text {mean }}=3.43$ ) in our task. Also consistent with previous fMRI investigations of error processing [for review, see Hester et al. (2004), Klein et al. (2007), and Taylor et al. (2007)], further error-related activation was evident in the bilateral anterior insula/frontal operculum (aI/fO) and the right LPFC, specifically in the inferior frontal junction (IFJ) (Fig. 3). A summary of activations, including those evident in the reverse correct $>$ error contrast, can be found in Table 1. 
Table 1. Error versus correct activation maxima

\begin{tabular}{|c|c|c|c|c|c|}
\hline Anatomical area & Hemisphere & $x$ & $y$ & $z$ & $Z_{\max }$ \\
\hline \multicolumn{6}{|l|}{ Error $>$ correct } \\
\hline pMFC & $\mathrm{L} / \mathrm{R}$ & 1 & 17 & 42 & 5.34 \\
\hline IFJ & $\mathrm{R}$ & 37 & 2 & 39 & 3.48 \\
\hline $\mathrm{al} / \mathrm{f0}$ & $\mathrm{L}$ & -35 & 20 & 6 & 5.21 \\
\hline $\mathrm{al} / \mathrm{f0}$ & $\mathrm{R}$ & 37 & 23 & 3 & 4.55 \\
\hline \multicolumn{6}{|l|}{ Correct $>$ error } \\
\hline Paracentral lobule & $\mathrm{L}$ & -20 & -37 & 60 & -3.14 \\
\hline Precentral gyrus/central sulcus & $\mathrm{R}$ & 43 & 28 & 57 & -3.49 \\
\hline Precentral gyrus/central sulcus & $\mathrm{L}$ & -44 & -19 & 54 & -2.88 \\
\hline Precuneus & $\mathrm{R}$ & 16 & -73 & 51 & -3.93 \\
\hline Superior frontal gyrus & $\mathrm{L}$ & -20 & 41 & 42 & -4.11 \\
\hline Posterior cingulate gyrus & $\mathrm{R}$ & 10 & -25 & 39 & -3.54 \\
\hline Posterior cingulate gyrus & $\mathrm{L}$ & -5 & -37 & 39 & -3.73 \\
\hline Superior occipital gyrus/cuneus** & $\mathrm{R}$ & 22 & -79 & 27 & -3.78 \\
\hline Dorsal striatum* & $\mathrm{L}$ & -18 & 2 & 24 & -3.99 \\
\hline Dorsal striatum* & $\mathrm{R}$ & 13 & -1 & 24 & -4.02 \\
\hline Thalamus (anterior) & $\mathrm{L} / \mathrm{R}$ & -5 & -12 & 18 & -3.83 \\
\hline Posterior cingulate gyrus/precuneus & $\mathrm{L}$ & -11 & -52 & 18 & -5.07 \\
\hline Putamen* & $\mathrm{L}$ & -23 & -7 & 12 & -4.10 \\
\hline Superior frontal gyrus & $\mathrm{L}$ & -23 & 62 & 9 & -4.01 \\
\hline Superior temporal gyrus & $\mathrm{R}$ & 55 & -13 & 9 & -3.73 \\
\hline Superior temporal gyrus & $\mathrm{L}$ & -40 & -40 & 9 & -4.43 \\
\hline Caudate nucleus* & $\mathrm{R}$ & 10 & 14 & 6 & -5.28 \\
\hline Middle occipital gyrus** & $\mathrm{R}$ & 43 & -73 & 6 & -4.11 \\
\hline Middle occipital gyrus** & $\mathrm{R}$ & 34 & -88 & 6 & -4.15 \\
\hline Superior temporal gyrus & $\mathrm{L}$ & -53 & -10 & 3 & -3.42 \\
\hline Perigenual cingulate & $\mathrm{R}$ & 13 & 38 & 6 & -3.51 \\
\hline Perigenual cingulate/gyrus rectus & $\mathrm{L}$ & -8 & 41 & -6 & -4.62 \\
\hline Ventral striatum* & $\mathrm{L}$ & -18 & 2 & -6 & -4.81 \\
\hline Ventral striatum* & $\mathrm{R}$ & 16 & 0 & -6 & -4.84 \\
\hline Posterior fusiform gyrus** & $\mathrm{R}$ & 34 & -70 & -6 & -3.84 \\
\hline Hippocampus* & $\mathrm{L}$ & -23 & -19 & -9 & -4.58 \\
\hline Posterior fusiform gyrus** & $\mathrm{L}$ & -29 & -79 & -9 & -4.31 \\
\hline Hippocampus* & $\mathrm{R}$ & 25 & -16 & -12 & -4.21 \\
\hline Cerebellum** & $\mathrm{L} / \mathrm{R}$ & -2 & -70 & -15 & -4.47 \\
\hline
\end{tabular}

Talairach coordinates correspond to activation peaks of independent clusters $\left(Z_{\text {mean }} \geq 2.58\right)$ individually composed of $\geq 23$ voxels ( $623 \mathrm{~mm}^{3} ; p<0.05$; corrected) and separated by a minimum $15 \mathrm{~mm}$ radius. Asterisk indicate clusters within greater activations that exceeded $20,000 \mathrm{~mm}^{3}$ (741 voxels): ${ }^{*}$ continuous subcortical cluster, ${ }^{*}$ continuous posterior cluster. R, Right; $L$, left.
A number of studies have found relationships between errorrelated pMFC activity and both PES (Gehring et al., 1993; Kerns et al., 2004; Debener et al., 2005; Klein et al., 2007; Cavanagh et al., 2009) and PERI (de Bruijn et al., 2004; Ridderinkhof et al., 2002), but findings have been inconsistent (Gehring and Fencsik, 2001; Carp and Compton, 2009; Núñez Castellar et al., 2010), most notably in patients with pMFC damage (Fellows and Farah, 2005). To explore whether error-related activity in this region was associated with post-error behavior in our task, we conducted group analyses of the individual error $>$ correct activation patterns in the empirically defined error-sensitive pMFC (Fig. 3, Table 1) informed with the individual PES and PERI covariates (Materials and Methods). Results revealed a relationship between error-related hemodynamic activity in the rostral cingulate zone (RCZ) and PES and tentative evidence that the pre-supplementary motor area (pre-SMA) may mediate PERI (supplemental Fig. S1, available at www.jneurosci.org as supplemental material).

\section{Post-error control adjustments}

Currently influential views of performance monitoring and reinforcement learning propose that the functional significance of error-related pMFC activity is to guide cognitive control mechanisms needed to reinstate goal-directed behavior (Botvinick et al., 2001; Holroyd and Coles, 2002; Ridderinkhof et al., 2004; Ullsperger and von Cramon, 2004; Yeung et al., 2004; Brown and Braver, 2005; Holroyd et al., 2005). In line with these hypotheses and previous investigations of PEBAs (Garavan et al., 2002; Kerns et al., 2004; Hester et al., 2007; Marco-Pallarés et al., 2008; West and Travers, 2008; Cavanagh et al., 2009), an analysis of posterror $>$ correct BOLD activity revealed elevated activity in regions commonly associated with cognitive control and theoretically conducive to restoration of successful performance in our task. Specifically, increased activation was evident in the left anterior prefrontal cortex (aPFC) $(x=-35, y=53, z=15 ; 675$ $\left.\mathrm{mm}^{3}, Z_{\text {mean }}=2.90\right)$, implicated for example in voluntary reallocation of attention (Pollmann, 2004), and the right inferior parietal lobule (IPL) $\left(x=37, y=-55, z=45 ; 1215 \mathrm{~mm}^{3}, Z_{\text {mean }}=2.91\right)$
A

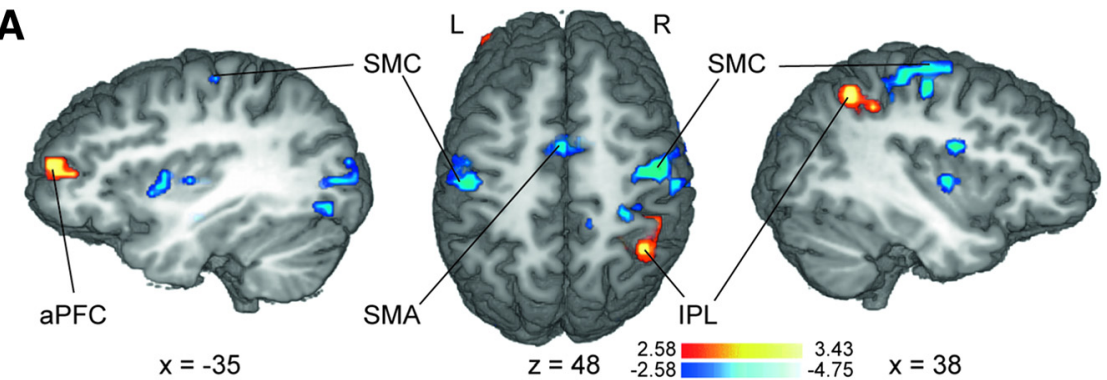

B
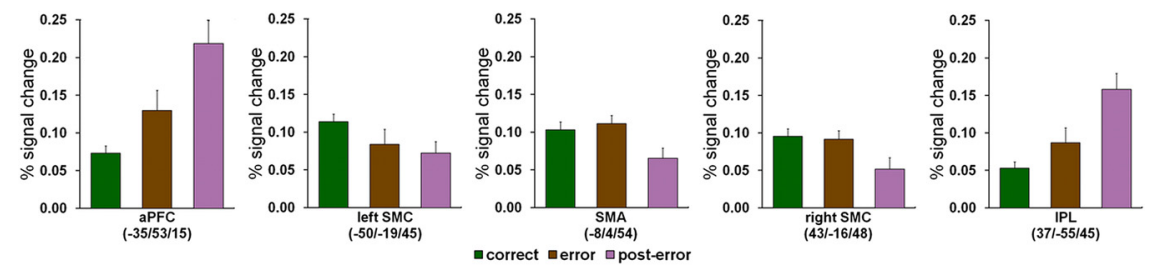

Figure 4. Activation patterns in brain regions associated with post-error control adjustments. $\boldsymbol{A}$, Multiple-comparisons corrected ( $p<0.05$ ) Z-maps contrasting the conditions post-error versus correct are plotted on selected slices of a 3D-rendered individual brain in Talairach space. Warm colors indicate greater activity on post-error trials relative to correct trials, while cool colors indicate the reverse pattern. R, Right; $L$, left. $B$, Group mean BOLD percentage signal change on correct, error, and post-error trials ( \pm SEM) in selected regions associated with post-error control adjustments.
(Fig. 4), recently shown to be involved in early visuomotor integration in the Simon task (Stürmer et al., 2007). Although these findings suggest increased cognitive control following errors, demonstration of control adjustments also requires that neural activity in brain regions specialized in processing information crucial to meeting task-specific demands is biased in a goal-directed manner (Miller and Cohen, 2001).

Given the demands of our task, we expected that control adjustments following errors would serve to either (1) suppress inappropriate response activation primed by irrelevant spatial stimulus features (Praamstra et al., 1999; Stürmer et al., 2002; Stürmer and Leuthold, 2003) and/or (2) enhance sensory processing of relevant face features. Indicative of increased control over response-related processing following errors, the reverse correct $>$ post-error contrast revealed reduced post-error activity in medial motor structures extending from the supplementary motor area (SMA; local 
Table 2. Post-error versus correct activation maxima

\begin{tabular}{llrrrr}
\hline Anatomical area & Hemisphere & $x$ & $y$ & $z$ & $Z_{\max }$ \\
\hline $\begin{array}{llrrr}\text { Post-error }>\text { correct } \\
\text { IPL }\end{array}$ & $\mathrm{L}$ & 37 & -55 & 45 & 3.43 \\
$\quad$ aPFC & $\mathrm{L}$ & -35 & 53 & 15 & 3.41 \\
Correct $>$ post-error & & & & & \\
$\quad$ Paracentral lobule* & $\mathrm{L} / \mathrm{R}$ & -5 & -34 & 57 & -4.62 \\
SMA* & $\mathrm{L} / \mathrm{R}$ & -8 & -4 & 54 & -4.61 \\
Postcentral gyrus/central sulcus & $\mathrm{R}$ & 31 & -31 & 54 & -4.03 \\
Precentral gyrus/central sulcus & $\mathrm{R}$ & 43 & -16 & 48 & -4.75 \\
Precentral gyrus/central sulcus & $\mathrm{L}$ & -50 & -19 & 45 & -3.54 \\
Mid-insula & $\mathrm{R}$ & 34 & -4 & 21 & -4.47 \\
Parieto-occipital sulcus & $\mathrm{R}$ & 19 & -67 & 21 & -3.51 \\
Postcentral gyrus & $\mathrm{R}$ & 61 & -21 & 18 & -4.37 \\
Middle occipital gyrus & $\mathrm{L}$ & -35 & -88 & 18 & -3.99 \\
Putamen (posterior) & $\mathrm{L}$ & -29 & -13 & 9 & -3.27 \\
Superior temporal gyrus & $\mathrm{L}$ & -47 & -28 & 9 & -4.17 \\
Middle occipital gyrus & $\mathrm{L}$ & -41 & -73 & 6 & -3.93 \\
Putamen (anterior) & $\mathrm{L}$ & -32 & 5 & 3 & -3.36 \\
Mid-insula & $\mathrm{R}$ & 40 & -4 & 3 & -3.46 \\
Perigenual cingulate & $\mathrm{L} / \mathrm{R}$ & -11 & 29 & -3 & -3.61 \\
Middle occipital gyrus & $\mathrm{L}$ & -53 & -64 & -6 & -3.19 \\
Posterior fusiform gyrus & $\mathrm{L}$ & -20 & -82 & -6 & -4.13 \\
Hippocampus & $\mathrm{L}$ & -29 & -16 & -9 & -3.58 \\
\hline
\end{tabular}

Talairach coordinates correspond to activation peaks of independent clusters $\left(Z_{\text {mean }} \geq 2.58\right)$ individually composed of $\geq 20$ voxels $\left(540 \mathrm{~mm}^{3} ; p<0.05\right.$; corrected) and separated by a minimum $15 \mathrm{~mm}$ radius. ${ }^{*}$ Clusters within greater activations that exceeded $5000 \mathrm{~mm}^{3}$ (185 voxels). R, Right; L, left.

peak: $\left.x=-8, y=-4, z=54 ; Z_{\max }=-4.61\right)$ to the paracentral lobule $\left(x=-5, y=-34, z=57 ; 5931 \mathrm{~mm}^{3} ; Z_{\text {mean }}=-3.10\right)$ and, most notably, bilateral regions of SMC (left, $x=-50, y=-19, z=$ $45 ; 1782 \mathrm{~mm}^{3} ; Z_{\text {mean }}=-2.93$; right, $x=43, y=-16, z=48 ; 3672$ $\mathrm{mm}^{3} ; Z_{\text {mean }}=-3.09$ ) (Fig. 4). Although it is unclear whether suppressed post-error SMA activity reflected control over eye movements, index finger responses, or another more complex condition-action association attributed to this region (cf. Nachev et al., 2008), the lateral SMC clusters, which corresponded to the motor hand areas, are suggestive of inhibitory control (Aron, 2007) over potentially inappropriate manual responses (i.e., increased motor response threshold). All activations evident in the post-error versus correct analyses are summarized in Table 2.

To test whether post-error control adjustments operated to bias sensory processing in favor of task-relevant information, we submitted the BOLD signal change data recorded in the individually defined FFA ROIs (Materials and Methods) (Fig. 5A) to 2 (trial $n-1$ accuracy) $\times 2$ (trial $n$ compatibility) repeatedmeasures ANOVAs. Thus, we could control for the possibility that the hypothesized post-error versus correct differences in target-feature processing were qualified by variation in activation attributable to cognitive control dynamics elicited by conflict processing (Botvinick et al., 2001; Egner and Hirsch, 2005). As predicted, FFA activation was elevated on post-error trials in both the left $\left(F_{(1,20)}=5.9 ; p=0.03\right)$ and right $\left(F_{(1,20)}=7.0 ; p=0.02\right)$ hemispheres (Fig. $5 B$ ). Although neither ROI showed sensitivity to S-R compatibility (both $F_{(1,20)}<1.8$; both $p>0.2$ ) and no interactions were present (both $F_{(1,20)}<1.1$; both $p>0.3$ ), it is important to note that FFA activation was elevated already on error versus correct trials, but only in the right hemisphere $\left(t_{(20)}=\right.$ $2.8 ; p=0.01)$ and not in the left $\left(t_{(20)}=0.7 ; p>0.5\right)$. One possible explanation for increased FFA activity on error trials that is consistent with recent electrophysiological data (Cohen et al., 2009) is that online control adjustments redirect attention to relevant information during or immediately following response execution, but do not produce sensory representations strong enough cancel the ongoing incorrect response.
A

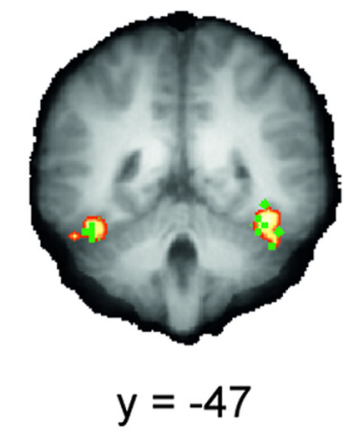

3.09

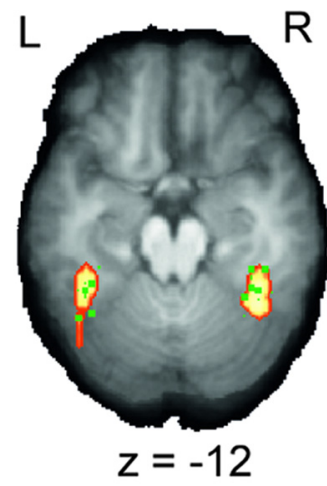

5.89

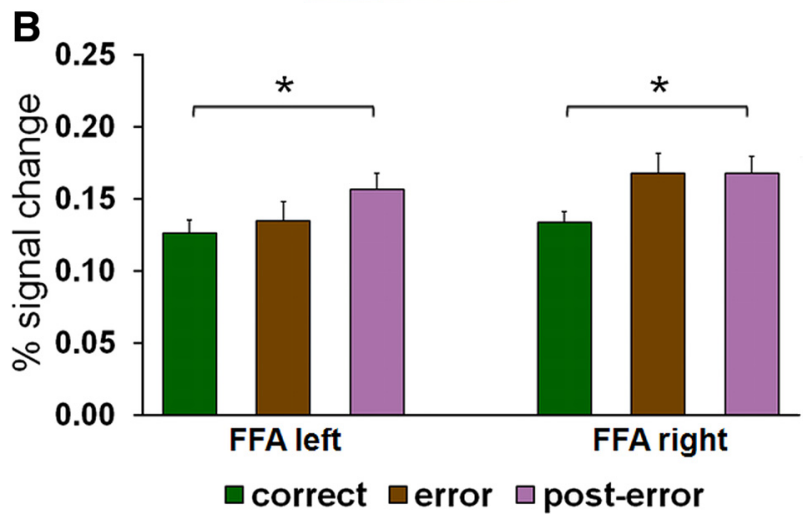

Figure 5. Post-error enhancement of task-relevant sensory processing. $\boldsymbol{A}$, Face-preferential activation in the left $\left(x=-38 \pm 2, y=-46 \pm 8, z=-12 \pm 6 ; 1701 \mathrm{~mm}^{3} ; z_{\text {mean }}=3.60\right)$ and right $\left(x=37 \pm 4, y=-46 \pm 5, z=-12 \pm 5 ; 2025 \mathrm{~mm}^{3} ; Z_{\text {mean }}=3.91\right)$ fusiform gyrus revealed by a group analysis of the FFA localizer task data contrasting face versus scrambled face stimuli is plotted on selected slices of the sample mean brain at $p<0.001$, corrected ( \pm refers to the range of peak FFA Talairach coordinates identified in individual participants). Green points correspond to the location of the individually defined FFA ROIs visible in the shown slices. $\boldsymbol{B}$, Group mean BOLD percentage signal change averaged for correct, error, and post-error trials ( \pm SEM) measured in the individual FFA ROls. ${ }^{*} p<0.05$.

Additional analyses of FFA activity excluded the likelihood that increased post-error activation was driven by bottom-up effects attributable to a neural preference for faces presented in the contralateral visual field (Hemond et al., 2007) (supplemental Fig. S2, available at www.jneurosci.org as supplemental material). Furthermore, indicating that post-error modulation of sensory processing was not likely a generic effect in visual cortex, control analyses in empirically defined regions of the ventral visual stream (Materials and Methods) and location-sensitive early visual cortex revealed no activation differences on post-error versus correct trials (supplemental Fig. S2, available at www.jneurosci.org as supplemental material). Interestingly, however, although we did not find clear evidence of distractor-feature inhibition in sensory cortex (Kastner and Ungerleider, 2000; Polk et al., 2008; Weissman et al., 2009) following errors, we uncovered significant post-error reductions in lateralization of BOLD responses driven by task-irrelevant spatial information in both hemispheres of location-sensitive early visual cortex (supplemental Fig. S2, available at www.jneurosci.org as supplemental material).

\section{Individual differences in PEBAs explain post-error motor suppression and sensory amplification}

We predicted that post-error control adjustments would not only be associated with modulation of lower-level task-related motor and/or sensory processing, but that such modulation would de- 

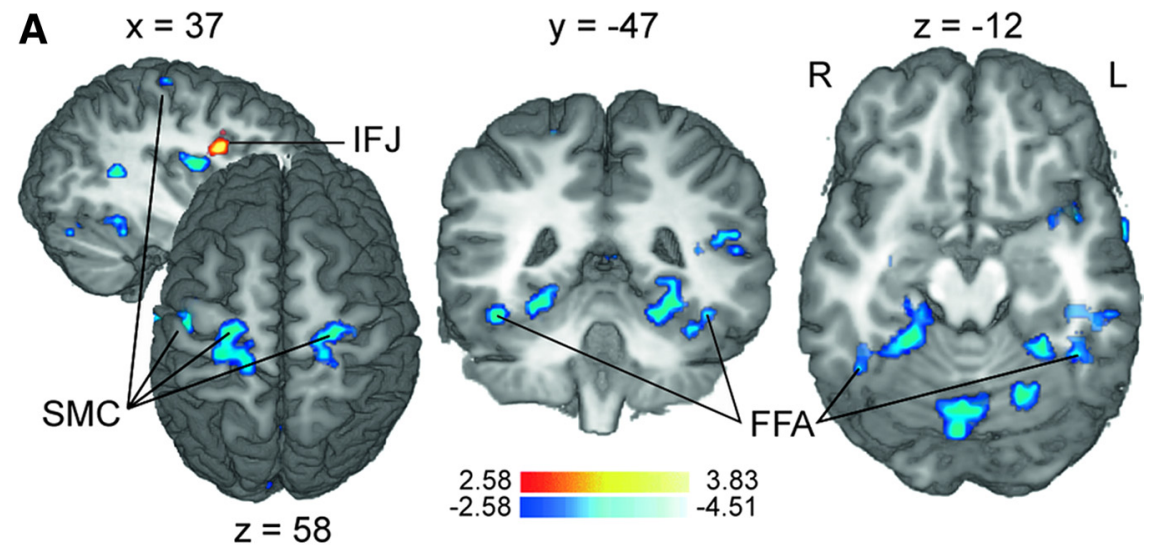

B
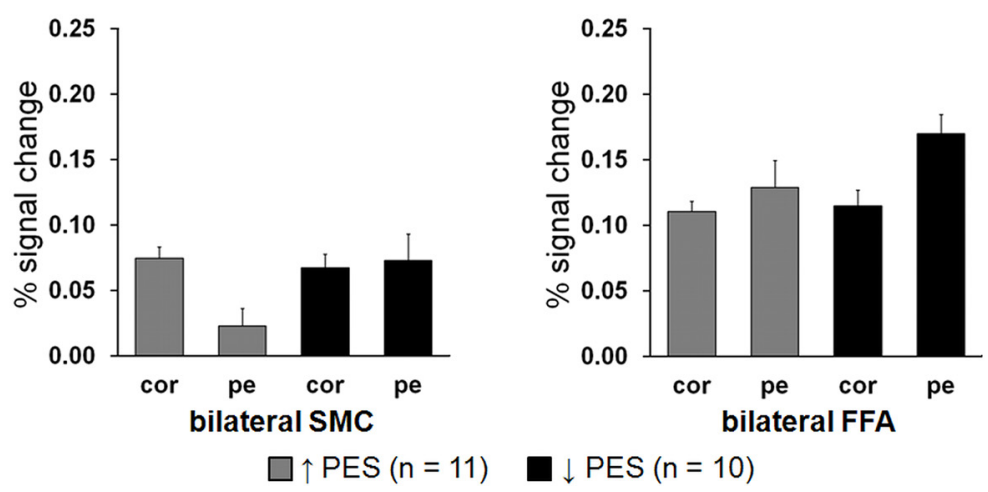

Figure 6. PES-dependent post-error activation. $\boldsymbol{A}$, PES-informed post-error $>$ correct activation is displayed at $p<0.05$ (corrected) on selected slices of a 3D-rendered individual brain in Talairach space. Warm colors indicate positive correlations between the post-error $>$ correct BOLD response and greater degrees of PES, while cool colors signify negative correlations. $R$, Right; $L$, left. B, Differential contributions of post-error slowing and post-error "speeding" to the negative correlations in the SMC and FFA evident in the PES-informed post $>$ correct analysis. Percentage BOLD signal change averaged from the identified bilateral SMC regions (left; $1836 \mathrm{~mm}^{3}$ ) and group average bilateral FFA (right; $3726 \mathrm{~mm}^{3}$ ) on correct and post-error trials are presented separately for participants who tended to show PES ("slowers") and those who tended to maintain/improve RTs following errors ("speeders") based on a median split of individual PES scores.

pend on how participants adjusted performance following errors. To address this hypothesis, we incorporated individual degrees of PES and PERI as parametric covariates in group analyses of the individual post-error $>$ correct activation patterns (Materials and Methods). Only one region displayed post-error activity positively related to the degree of PES: the right IFJ, partially corresponding to the same area activated on error $>$ correct trials $(x=$ $29, y=2, z=27 ; 513 \mathrm{~mm}^{3} ; Z_{\text {mean }}=-3.12$ ) (Fig. $6 \mathrm{~A}$; see also Fig. $3)$. In contrast, negative correlations between PES and post-error activity were evident in the same region of the left SMC identified by the overall correct $>$ post-error analysis $(x=-41, y=-16$, $\left.z=51 ; 567 \mathrm{~mm}^{3} ; Z_{\text {mean }}=-3.13\right)$ and somewhat more superior and posterior bilateral SMC regions (left: $x=-26, y=-34, z=$ $57 ; 1215 \mathrm{~mm}^{3} ; Z_{\text {mean }}=-2.92$; right: $x=19, y=-25, z=60 ; 621$ $\mathrm{mm}^{3} ; Z_{\text {mean }}=-2.93$ ) (Fig. $6 A$; see also Fig. 4). Thus, generally suppressed post-error SMC activity (Fig. 4) could be explained, at least partially, by greater PES. Equally important, negative correlations were also evident in ventral occipitotemporal cortex with significant voxels located within the bilateral group mean FFA (local peak left: $x=-41, y=-43, z=-12 ; Z_{\max }=-3.33$; right: $x=37, y=-49, z=-12 ; Z_{\max }=-2.94$ ) (Fig. $6 A$ ). Although the negative relationship between PES and post-error activity was not clearly reflected in the BOLD percentage signal change data recorded in the individually defined FFA ROIs, correlations were negative in both the left $(r=-0.41 ; p=0.06)$ and right $(r=$ $-0.34 ; p=0.13$ ) hemispheres. These particular findings seem to suggest that the more participants slowed down on post-error trials, the less they attended to faces. It is important to note, however, that a negative correlation between greater PES and post-error FFA activity can also indicate a positive correlation between that and less PES (i.e., maintaining/improving response speed following errors). Indeed, inspection of FFA activation based on a median split of post-error "slowers" and post-error "speeders" showed that FFA activity was notably increased on post-error trials in participants who tended to generally maintain/improve RTs following errors, but only negligibly modulated in those who tended to slow down. In contrast, consistent with the notion that PES may reflect an increase in the motor response threshold (Botvinick et al., 2001; Holroyd et al., 2005), post-error SMC activity was reduced in post-error "slowers," but not modulated in "speeders" (Fig. 6B). A complete list of PES-dependent post-error $>$ correct activation patterns is presented in Table 3.

An analog analysis of the individual post-error $>$ correct activation patterns informed with individual differences in the ability to reduce interference following errors (PERI) revealed positive correlations in the left LPFC, specifically in the superior frontal sulcus (SFS; $x=-14, y=$ $\left.35, z=42 ; 702 \mathrm{~mm}^{3} ; Z_{\text {mean }}=2.94\right)$, the left superior colliculus (SC; $x=-8, y=$ $\left.31, z=0 ; 567 \mathrm{~mm}^{3} ; Z_{\text {mean }}=2.94\right)$, and, most importantly, a region of the left fusiform gyrus $\left(x=-35, y=-43, z=-6 ; 1053 \mathrm{~mm}^{3} ; Z_{\text {mean }}=\right.$ $2.90)$ that partially overlapped the group average FFA volume (local peak: $x=-41, y=-49, z=-10 ; Z_{\max }=2.78$ ) (Fig. 7A). Positive correlations were also evident in the group average right FFA, but only at a less conservative significance threshold $(x=34$, $y=-46, z=-12 ; 216 \mathrm{~mm}^{3} ; Z_{\text {mean }}=2.54 ; p<0.01$; uncorrected). Supporting the findings in the FFA, positive relationships between greater degrees of PERI and the post-error BOLD signal change data were also reflected in both the individually defined left $(r=0.44 ; p=$ $0.04)$ and right $(r=0.65 ; p=0.001)$ FFA (Fig. $7 B)$. No regions showing negative correlations with individual differences in PERI reached significance.

\section{Discussion}

We used fMRI to investigate the hypothesis that PEBAs index adjustments in cognitive control (Ridderinkhof et al., 2004). Replicating previous investigations of PEBAs (Garavan et al., 2002; Kerns et al., 2004; Marco-Pallarés et al., 2008; Cavanagh et al., 2009), we found a characteristic pattern of error-related activation including the $\mathrm{pMFC}$ and post-error increases in activity in prefrontal and parietal control regions. Building on these studies, we showed that post-error control adjustments can bias lowerlevel task-related processing in a goal-directed manner. Specifically, in a task that required selective attention to gender features of faces while overriding response activation primed by taskirrelevant face location, we found both (1) suppression of poten- 
tially inappropriate motor responses (as reflected in decreased SMC activation) and (2) amplification of task-relevant sensory processing (as reflected in increased FFA activity). More importantly, we found that post-error modulation of motor and sensory processing was dependent on how participants adjusted performance on those trials. While participants who tended to show general response slowing following errors (PES) explained suppression of response-related SMC activity, those who tended to show a selective improvement in interference resolution (PERI) and/or maintain/improve response speed on post-error trials explained enhancement of target processing in the FFA. Together, these findings significantly advance our understanding of the neural mechanisms by which performance is adapted following errors.

\section{Post-error slowing}

PES is frequently thought to index a strategic control mechanism that prevents future errors by suppressing motor response activation (i.e., increasing the response threshold) (Botvinick et al., 2001; Holroyd et al., 2005). The negative correlation between PES and SMC activity revealed here delivers novel support for this hypothesis. However, although PES occasionally coincides with improved accuracy following errors (Rabbitt, 1966; Laming, 1968; Marco-Pallarés et al., 2008), we found no such relationship and several studies have even reported decreased post-error accuracy (Rabbitt and Rodgers, 1977; Laming, 1979; Hajcak and Simons, 2008). Therefore, alternative interpretations may need to be considered.

It has been suggested that PES may reflect persistence of a breakdown in processing that contributed to the error (Gehring and Fencsik, 2001) or errorevoked arousal that interferes with task preparation (Carp and Compton, 2009). A recent proposal explains PES as the consequence of an unspecific orienting response elicited by unexpected infrequent events (Notebaert et al., 2009) that "turns attention away from the task" and "delays processing of the following stimulus" (Núñez Castellar et al., 2010). Generally in line with these views, participants who showed greater PES in our task showed no noteworthy modulation of target processing in the FFA on post-error trials. Further, PES was associated with post-error activation of the same region of the right LPFC that was already activated on error trials: the IFJ, a region implicated in transient task-set updating (Brass et al., 2005; Derrfuss et al., 2005). Given the proximity of the IFJ to inferior frontal regions implicated in response inhibition (Aron et al., 2004) and premotor regions shown to mediate control over "direct" response activation in the Simon task (Praamstra et al., 1999; Egner et al., 2007), it is tempting to speculate a causal relationship between PES-dependent IFJ activation and suppression of SMC activity in our task. However, although the IFJ is often recruited in response inhibition tasks, its function in such contexts may be more related to an orienting response triggered by infrequent "No-Go" trials than to motor control per se
Table 3. PES-dependent post-error > correct activation maxima

\begin{tabular}{|c|c|c|c|c|c|}
\hline Anatomical area & Hemisphere & $x$ & $y$ & $z$ & $Z_{\max }$ \\
\hline Inferior frontal sulcus/IFJ & $\mathrm{R}$ & 29 & 2 & 27 & 3.83 \\
\hline Precentral gyrus/central sulcus & $\mathrm{R}$ & 19 & -25 & 60 & -3.55 \\
\hline $\begin{array}{l}\text { Postcentral gyrus/central sulcus/ } \\
\text { precentral gyrus }\end{array}$ & $\mathrm{L}$ & -26 & -34 & 57 & -3.48 \\
\hline Precentral gyrus/central sulcus & $\mathrm{L}$ & -41 & -16 & 51 & -3.69 \\
\hline Precentral gyrus & $\mathrm{L}$ & -59 & -13 & 30 & -3.65 \\
\hline Posterior insula & $\mathrm{L}$ & -35 & -19 & 21 & -3.32 \\
\hline Mid-insula & $\mathrm{R}$ & 31 & -7 & 15 & -4.01 \\
\hline Superior temporal gyrus** & L & -41 & -37 & 15 & -3.30 \\
\hline Superior temporal sulcus & $\mathrm{R}$ & 34 & -49 & 12 & -3.48 \\
\hline Calcarine sulcus & $\mathrm{L} / \mathrm{R}$ & 4 & -85 & 12 & -3.71 \\
\hline Thalamus (dorsal) & $\mathrm{R}$ & 4 & -22 & 9 & -4.02 \\
\hline Mid-insula & $\mathrm{L}$ & -38 & 5 & -3 & -4.51 \\
\hline Collateral sulcus/fusiform gyrus & $\mathrm{L}$ & -29 & -49 & -9 & -4.00 \\
\hline Inferior temporal/fusiform gyrus & $\mathrm{L}$ & -50 & -37 & -12 & -3.63 \\
\hline $\mathrm{FFA}^{*}$ & $\mathrm{~L}$ & -41 & -43 & -12 & -3.33 \\
\hline Collateral sulcus/fusiform gyrus & $\mathrm{R}$ & 19 & -43 & -12 & -3.90 \\
\hline $\mathrm{FFA}^{*}$ & L & 37 & -49 & -12 & -2.94 \\
\hline Cerebellum** & $\mathrm{L} / \mathrm{R}$ & -5 & -67 & -15 & -3.61 \\
\hline Cerebellum ${ }^{* *}$ & $\mathrm{~L}$ & -23 & -64 & -15 & -3.59 \\
\hline Cerebellum** & $\mathrm{R}$ & 22 & -67 & -15 & -3.32 \\
\hline Midbrain/amygdala & $\mathrm{L}$ & -11 & -22 & -15 & -4.20 \\
\hline Amygdala & $\mathrm{R}$ & 22 & -7 & -18 & -3.58 \\
\hline
\end{tabular}

Talairach coordinates correspond to activation peaks of independent clusters $\left(Z_{\text {mean }} \geq 2.58\right)$ individually comprised of $\geq 18$ voxels ( $486 \mathrm{~mm}^{3} ; p<0.05$; corrected) and separated by a minimum $15 \mathrm{~mm}$ radius. *Local maximum within the group average FFA volume. **Clusters within greater activations that exceeded $3000 \mathrm{~mm}^{3}$ (111 voxels). R, Right; L, left.

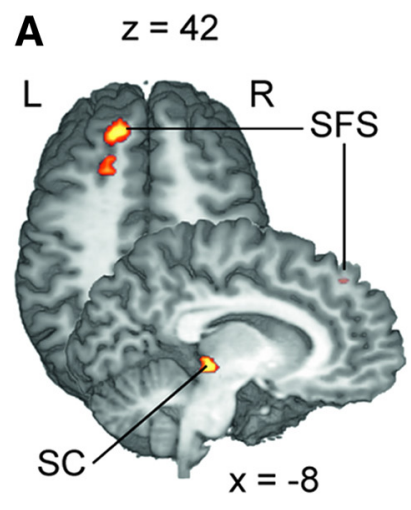

B

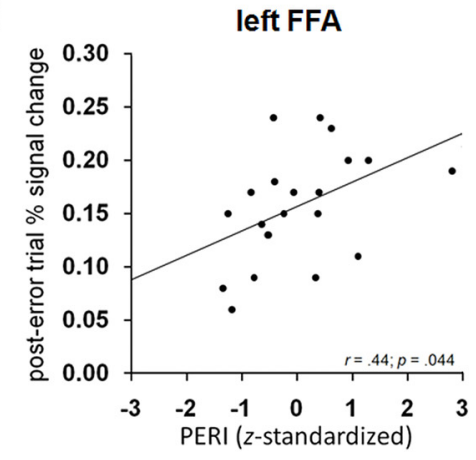

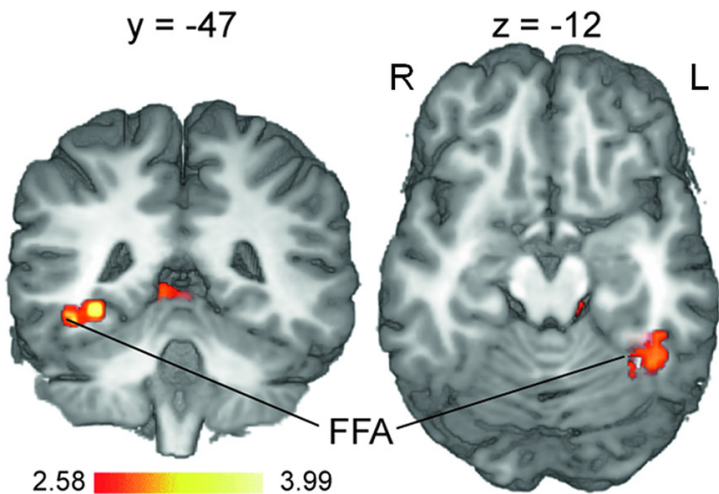

right FFA

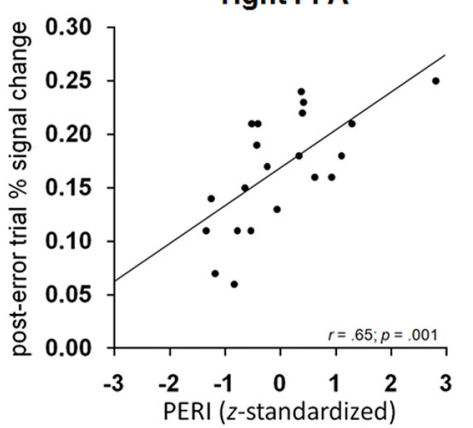

Figure 7. PERI-dependent post-error activation. $\boldsymbol{A}$, PERI-informed post-error $>$ correct activation is displayed at $p<0.05$ (corrected) on selected slices of a 3D-rendered individual brain in Talairach space. Warm colors indicate positive correlations between the post-error $>$ correct BOLD response and greater degrees of PERI. R, Right; L, left. B, Correlations between post-error BOLD activity and PERI in the individually defined FFA. Each point corresponds to the averaged data from one participant.

(Chikazoe et al., 2009). Overall, these results suggest that PES may not have reflected deliberate response cautiousness directed at avoiding errors in our task, but rather the consequence of an orienting response that interferes with task preparation and stim- 
ulus processing, resulting in the need to suppress potentially incorrect responses activated by irrelevant spatial stimulus features when attention fails to bias sensory processing in favor of relevant features.

\section{Post-error reduction of interference}

PERI has been proposed to reflect a "selective suppression of response activation" - a specific inhibitory control mechanism (Ridderinkhof, 2002). Based on this hypothesis and previous findings that behavioral adaptation in the Simon task is achieved by biasing response channels (Stürmer et al., 2002) rather than sensory pathways (Stürmer and Leuthold, 2003), one might expect that PERI would be expressed as motor control in our task. Instead, we found improved interference resolution following errors to be associated with enhancement of task-relevant sensory processing in the FFA. Although enhanced target processing may have been achieved by rapid reallocation of attentional resources following stimulus presentation on the post-error trials, it seems more plausible, given the brief duration of stimulus presentation (150 ms), that PERI-dependent FFA modulation was driven primarily by prestimulus biasing of sensory pathways. This view implies that participants who showed greater PERI maintained relevant representations during the post-error ISI. One possibility is that the region of the left LPFC found to mediate PERI (SFS) held relevant representations online following errors, thereby enabling efficient target selection upon stimulus presentation. Supporting this interpretation, a recent transcranial magnetic stimulation study using a Simon task showed that between-trial behavioral adaptation was impaired by interruption of left LPFC processing in anticipation of the upcoming stimulus (Stürmer et al., 2007). Nonetheless, given that spatial attention to irrelevant spatial stimulus features was more or less imperative in our task, some degree of inhibitory control may have been required to reduce interference following errors. We speculate that PERI-dependent activation of the SC, a region known to exert control over eye movements, may have reflected covert spatial attention (Müller et al., 2005) — that is, withholding saccades toward the source of stimulation, thereby facilitating response selection by minimizing the weight attention assigns to irrelevant spatial features.

\section{PES and PERI: dual control mechanisms?}

The neural architecture(s) supporting sequential behavioral adaptation to conflicts in information processing [of which errors are thought to be a special case (Yeung et al., 2004)] have been suggested to be determined by task-specific demands on lowerlevel processing (Egner, 2007, 2008; Egner et al., 2007). However, the dissociations between PES and PERI revealed here seem to speak to an alternative hypothesis-namely, that cognitive control may be primarily determined by when, and consequently how, it is executed by the individual when adjustments are needed: reactively or proactively (De Pisapia and Braver, 2006; Braver et al., 2007). According to this view, cognitive control varies depending on whether it is "reactive" in nature, directed at resolving interference ex post facto in a transient manner, or "proactive," focused on preventing interference ex ante facto in a preparatory fashion. Importantly, whereas the distinction of Braver et al. (2007) between dual control mechanisms is based largely on observations on a short- (e.g., event-related) versus long- (e.g., block-related) term time scale, our findings are more consistent with the conceptualization of Ridderinkhof et al. (2010) of reactive within-trial "online action control" and proactive between-trial "anticipatory regulation of online action con- trol." As noted above, PES seems to reflect an orienting response that impairs task preparation, necessitating a reactive "late correction" control mechanism following stimulus presentation on post-error trials - that is, after irrelevant information has begun to interfere with response selection. In contrast, PERI seems to index a proactive control mechanism characterized by preparatory task-set maintenance that enables "early selection" of relevant information, thereby reducing the influence of irrelevant information on response selection.

Critically, given the poor temporal resolution of the BOLD response, future research is needed to validate the interpretation that PES and PERI may differentially index "late correction" and "early selection" control mechanisms. Further, it should be emphasized that this interpretation is based solely on our observations of between-subjects variation in PEBAs in our particular task. Within subjects, it is conceivable that some errors will elicit an orienting response that allows the individual to be responsive to any potentially relevant stimulus, but others might trigger a proactive control mode that selectively primes relevant representations. An intriguing possibility is that different types of conflict differentially evoke reactive and proactive control adjustments. In any event, the finding that sequential behavioral adaptation in the Simon task can occur not only by biasing response channels (Stürmer et al., 2002; Egner et al., 2007), but also by biasing sensory pathways, supports the notion that the effects of cognitive control on task-related processing may not be determined primarily by task-specific demands (Egner et al., 2007; Egner, 2008), but can vary dramatically depending on how it is executed (Braver et al., 2007).

An important question is how adjustments such as those investigated here might be initiated. We found tentative evidence that distinct error-sensitive pMFC regions were differentially related to PES (RCZ) and PERI (pre-SMA). Although these relationships were not clear-cut, it is interesting to note that the precise constellation of our observations is consistent with metaanalytic data (Ridderinkhof et al., 2004), theoretical considerations (Rushworth, 2008; Hikosaka and Isoda, 2010), and recent empirical findings (Orr and Weissman, 2009) that suggest that the role of the RCZ in cognitive control may be more global in nature, while that of the pre-SMA may be more task specific.

\section{Conclusions}

In sum, the current study confirms relationships between PEBAs and cognitive control-that is, goal-directed modulation of lower-level task-related processing. Our data suggest that PES reflects an orienting response that necessitates a reactive, online control mechanism to suppress the influence of irrelevant information on motor output, while PERI seems to index a proactive control mechanism that selectively primes processing of relevant sensory input in a preparatory manner. To validate this interpretation, we are currently collecting electroencephalographic data with the same task. Further avenues of investigation might include determining whether the results obtained here generalize to other tasks and what subject and task variables might promote general versus specific adaptation effects.

\section{References}

Aron AR (2007) The neural basis of inhibition in cognitive control. Neuroscientist 13:214-228.

Aron AR, Robbins TW, Poldrack RA (2004) Inhibition and the right inferior frontal cortex. Trends Cogn Sci 8:170-177.

Botvinick MM, Braver TS, Barch DM, Carter CS, Cohen JD (2001) Conflict monitoring and cognitive control. Psychol Rev 108:624-652.

Brass M, Derrfuss J, Forstmann B, von Cramon DY (2005) The role of the 
inferior frontal junction area in cognitive control. Trends Cogn Sci 9:314-316.

Braver TS, Gray JR, Burgess GC (2007) Explaining the many varieties of working memory variation: dual mechanisms of cognitive control. In: Variation in working memory (Conway ARA, Jarrold C, Kane MJ, Miyake A, Towse JN, eds), pp 76-106. Oxford: Oxford UP.

Brown JW, Braver TS (2005) Learned predictions of error likelihood in the anterior cingulate cortex. Science 307:1118-1121.

Carp J, Compton RJ (2009) Alpha power is influenced by performance errors. Psychophysiology 46:336-343.

Carter CS, van Veen V (2007) Anterior cingulate cortex and conflict detection: an update of theory and data. Cogn Affect Behav Neurosci 7:367-379.

Cavanagh JF, Cohen MX, Allen JJ (2009) Prelude to and resolution of an error: EEG phase synchrony reveals cognitive control dynamics during action monitoring. J Neurosci 29:98-105.

Chikazoe J, Jimura K, Asari T, Yamashita K, Morimoto H, Hirose S, Miyashita Y, Konishi S (2009) Functional dissociation in right inferior frontal cortex during performance of go/no-go task. Cereb Cortex 19:146-252.

Cohen MX, van Gaal S, Ridderinkhof KR, Lamme VAF (2009) Unconscious errors enhance prefrontal-occipital oscillatory synchrony. Front Hum Neurosci 3:54.

Debener S, Ullsperger M, Siegel M, Fiehler K, von Cramon DY, Engel AK (2005) Trial-by-trial coupling of concurrent electroencephalogram and functional magnetic resonance imaging identifies the dynamics of performance monitoring. J Neurosci 25:11730-11737.

de Bruijn ERA, Hulstijn W, Verkes RJ, Ruigt GSF, Sabbe BGC (2004) Druginduced stimulation and suppression of action monitoring in healthy volunteers. Psychopharmacology 177:151-160.

De Pisapia N, Braver TS (2006) A model of dual control mechanisms through anterior cingulate and prefrontal cortex interactions. Neurocomputing 69:1322-1326.

Derrfuss J, Brass M, Neumann J, von Cramon DY (2005) Involvement of the inferior frontal junction in cognitive control: meta-analyses of switching and Stroop studies. Hum Brain Mapp 25:22-34.

Egner T (2007) Congruency sequence effects and cognitive control. Cogn Affect Behav Neurosci 7:380-390.

Egner T (2008) Multiple conflict-driven control mechanisms in the human brain. Trends Cogn Sci 12:374-380.

Egner T, Hirsch J (2005) Cognitive control mechanisms resolve conflict through cortical amplification of task-relevant information. Nat Neurosci 8:1784-1790.

Egner T, Delano M, Hirsch J (2007) Separate conflict-specific cognitive control mechanisms in the human brain. Neuroimage 35:940-948.

Fellows LK, Farah MJ (2005) Is anterior cingulate cortex necessary for cognitive control? Brain 128:788-796.

Garavan H, Ross TJ, Murphy K, Roche RA, Stein EA (2002) Dissociable executive functions in the dynamic control of behavior: inhibition, error detection, and correction. Neuroimage 17:1820-1829.

Gehring WJ, Fencsik DE (2001) Functions of the medial prefrontal cortex in the processing of conflict and errors. J Neurosci 21:9430-9437.

Gehring WJ, Goss B, Coles MGH, Meyer DE, Donchin E (1993) A neural system for error-detection and compensation. Psychol Sci 4:385-390.

Hajcak G, Simons RF (2008) Oops, I did it again: an ERP investigation of double-errors and action monitoring. Brain Cogn 68:15-21.

Hemond CC, Kanwisher NG, Op de Beeck HP (2007) A preference for contralateral stimuli in human object- and face-selective cortex. PLoS ONE 2:e574.

Hester R, Fassbender C, Garavan H (2004) Individual differences in error processing: a review and reanalysis of three event-related fMRI studies using the GO/NOGO task. Cereb Cortex 14:986-994.

Hester R, Barre N, Mattingley JB, Foxe JJ, Garavan H (2007) Avoiding another mistake: error and posterror neural activity associated with adaptive posterror behavior change. Cogn Affect Behav Neurosci 7:317-326.

Hikosaka O, Isoda M (2010) Switching from automatic to controlled behavior: cortico-basal ganglia mechanisms. Trends Cogn Sci 14:154-161.

Holroyd CB, Coles MG (2002) The neural basis of human error processing: reinforcement learning, dopamine, and the error-related negativity. Psychol Rev 109:679-709.

Holroyd CB, Yeung N, Coles MG, Cohen JD (2005) A mechanism for error detection in speeded response time tasks. J Exp Psychol Gen 134:163-191.

Jäger T, Seiler KH, Mecklinger A (2005) Picture database of morphed faces (MoFa): technical report. Psydoc Online. Retrieved June 26, 2010 from http://scidok.sulb.uni-saarland.de/volltexte/2005/453/.

Jentzsch I, Leuthold H (2006) Control over speeded actions: a common processing locus for micro- and macro-tradeoff effects? Q J Exp Psychol (Colchester) 59:1329-1337.

Kanwisher N, McDermott J, Chun MM (1997) The fusiform face area: a module in human extrastriate cortex specialized for face perception. J Neurosci 17:4302-4311.

Kastner S, Ungerleider L (2000) Mechanisms of visual attention in the human cortex. Annu Rev Neurosci 23:315-341.

Kerns JG, Cohen JD, MacDonald AW 3rd, Cho RY, Stenger VA, Carter CS (2004) Anterior cingulate conflict monitoring and adjustments in control. Science 303:1023-1026.

Klein TA, Endrass T, Kathmann N, Neumann J, von Cramon DY, Ullsperger M (2007) Neural correlates of error awareness. Neuroimage 34:17741781.

Kornblum S, Hasbroucq T, Osman A (1990) Dimensional overlap: cognitive basis for stimulus-response compatibility-a model and taxonomy. Psychol Rev 97:253-270.

Laming D (1979) Choice reaction performance following an error. Acta Psychol 43:199-224.

Laming DR (1968) Information theory of choice-reaction times. London: Academic.

Lohmann G, Müller K, Bosch V, Mentzel H, Hessler S, Chen L, Zysset S, von Cramon DY (2001) LIPSIA-a new software system for the evaluation of functional magnetic resonance images of the human brain. Comput Med Imaging Graph 25:449-457.

Lu CH, Proctor RW (1995) The influence of irrelevant location information on performance- a review of the Simon and spatial Stroop effects. Psychon Bull Rev 2:174-207.

Marco-Pallarés J, Camara E, Münte TF, Rodríguez-Fornells A (2008) Neural mechanisms underlying adaptive actions after slips. J Cogn Neurosci 20:1595-1610.

Miller EK, Cohen JD (2001) An integrative theory of prefrontal cortex function. Annu Rev Neurosci 24:167-202.

Müller JR, Philiastides MG, Newsome WT (2005) Microstimulation of the superior colliculus focuses attention without moving the eyes. Proc Natl Acad Sci U S A 102:524-529.

Nachev P, Kennard C, Husain M (2008) Functional role of the supplementary and pre-supplementary motor areas. Nat Rev Neurosci 9:856-869.

Notebaert W, Houtman F, Opstal FV, Gevers W, Fias W, Verguts T (2009) Post-error slowing: an orienting account. Cognition 111:275-279.

Núñez Castellar E, Kühn S, Fias W, Notebaert W (2010) Outcome expectancy and not accuracy determines posterror slowing: ERP support. Cogn Affect Behav Neurosci 10:270-278.

Orr JM, Weissman DH (2009) Anterior cingulate cortex makes 2 contributions to minimizing distraction. Cereb Cortex 19:703-711.

Polk TA, Drake RM, Jonides JJ, Smith MR, Smith EE (2008) Attention enhances the neural processing of relevant features and suppresses the processing of irrelevant features in humans: a functional magnetic resonance imaging study of the Stroop task. J Neurosci 28:13786-13792.

Pollmann S (2004) Anterior prefrontal cortex contributions to attention control. Exp Psychol 51:270-278.

Praamstra P, Kleine BU, Schnitzler A (1999) Magnetic stimulation of the dorsal premotor cortex modulates the Simon effect. Neuroreport 10: 3671-3674.

Proctor RW, Vu K-PL (2006) Stimulus-response compatibility: data, theory, and application. Boca Raton, FL: CRC.

Rabbitt P, Rodgers B (1977) What does a man do after he makes an error-an analysis of response programming. Q J Exp Psychol 29:727-743.

Rabbitt PM (1966) Errors and error correction in choice-response tasks. J Exp Psychol 71:264-272.

Ridderinkhof KR (2002) Micro- and macro-adjustments of task set: activation and suppression in conflict tasks. Psychol Res 66:312-323.

Ridderinkhof KR, de Vlugt Y, Bramlage A, Spaan M, Elton M, Snel J, Band GPH (2002) Alcohol consumption impairs detection of performance errors in mediofrontal cortex. Science 298:2209-2211.

Ridderinkhof KR, Ullsperger M, Crone EA, Nieuwenhuis S (2004) The role of the medial frontal cortex in cognitive control. Science 306:443-447.

Ridderinkhof KR, Forstmann BU, Wylie SA, Burle B, van den Wildenberg 
WPM (2010) Neurocognitive mechanisms of action control: resisting the call of the sirens. Wiley Interdiscip Rev Cogn Neurosci. Advance online publication. Retrieved July 17, 2010. doi:10.1002/wcs.99.

Rushworth MF (2008) Intention, choice, and the medial frontal cortex. Ann N Y Acad Sci 1124:181-207.

Rushworth MF, Buckley MJ, Behrens TE, Walton ME, Bannerman DM (2007) Functional organization of the medial frontal cortex. Curr Opin Neurobiol 17:220-227.

Simon JR (1969) Reactions toward the source of stimulation. J Exp Psychol $81: 174-176$.

Simon JR (1990) The effects of an irrelevant directional cue on human information processing. In: Stimulus-response compatibility (Proctor RW, Reeve TG eds), pp 31-86. Amsterdam: Elsevier.

Stürmer B, Leuthold H (2003) Control over response priming in visuomotor processing: a lateralized event-related potential study. Exp Brain Res 153:35-44.

Stürmer B, Leuthold H, Soetens E, Schröter H, Sommer W (2002) Control over location-based response activation in the Simon task: behavioral and electrophysiological evidence. J Exp Psychol Hum Percept Perform 28: $1345-1363$.

Stürmer B, Redlich M, Irlbacher K, Brandt S (2007) Executive control over response priming and conflict: a transcranial magnetic stimulation study. Exp Brain Res 183:329-339.
Talairach J, Tournoux P (1988) Co-planar stereotaxic atlas of the human brain. New York: Thieme.

Tanji J, Hoshi E (2008) Role of the lateral prefrontal cortex in executive behavioral control. Physiol Rev 88:37-57.

Taylor SF, Stern ER, Gehring WJ (2007) Neural systems for error monitoring: recent findings and theoretical perspectives. Neuroscientist 13:160-172.

Ullsperger M, Szymanowski F (2004) ERP correlates of error relevance. In: Errors, conflicts, and the brain. Current opinions on performance monitoring (Ullsperger M, Falkenstein M, eds), pp 171-177. Leipzig: MPI for Human Cognitive and Brain Sciences.

Ullsperger M, von Cramon DY (2004) Neuroimaging of performance monitoring: error detection and beyond. Cortex 40:593-604.

Weissman DH, Warner LM, Woldorff MG (2009) Momentary reductions of attention permit greater processing of irrelevant stimuli. Neuroimage 48:609-615.

West R, Travers S (2008) Tracking the temporal dynamics of updating cognitive control: an examination of error processing. Cereb Cortex 18:1112-1124.

Worsley KJ, Liao CH, Aston J, Petre V, Duncan GH, Morales F, Evans AC (2002) A general statistical analysis for fMRI data. Neuroimage 15:1-15.

Yeung N, Botvinick MM, Cohen JD (2004) The neural basis of error detection: conflict monitoring and the error-related negativity. Psychol Rev 111:931-959. 